\title{
Eye Ointment in Single-dose Container
}

National Cancer Institute

\section{Source}

National Cancer Institute. Eye Ointment in Single-dose Container. NCI Thesaurus. Code C149513.

Medicinal product consisting of an eye ointment presented in a single-dose container. 